\title{
A generalization of the Hahn-Banach theorem
}

\author{
by Jolanta Plewnia (Kraków)
}

\begin{abstract}
If $C$ is a non-empty convex subset of a real linear space $E, p: E \rightarrow \mathbb{R}$ is a sublinear function and $f: C \rightarrow \mathbb{R}$ is concave and such that $f \leq p$ on $C$, then there exists a linear function $g: E \rightarrow \mathbb{R}$ such that $g \leq p$ on $E$ and $f \leq g$ on $C$. In this result of Hirano, Komiya and Takahashi we replace the sublinearity of $p$ by convexity.

N. Hirano, H. Komiya and W. Takahashi gave the following generalization of the well-known Hahn-Banach theorem (Theorem 1 of [2]):

If $p$ is a sublinear function on a linear space $E, C$ is a non-empty convex subset of $E$ and $f$ is a concave functional on $C$ such that $f \leq p$ on $C$, then there exists a linear functional $g$ on $E$ such that $f \leq g$ on $C$ and $g \leq p$ on $E$.

The main goal of this paper is to give a new version of the above theorem with "sublinear" replaced by "convex". This result can be derived from an abstract Hahn-Banach theorem due to Rodé [6] (cf. also König [4]) or from the Nikodem theorem [5].

Our proof, based on an idea from [2], is an application of a theorem of Fan (Lemma 1).

In the proof of the main theorem we shall use the following two lemmas.

LEMMA 1 (Fan). Let $X$ be a non-empty compact convex subset of a Hausdorff linear topological space and $\left\{f_{\nu}: \nu \in I\right\}$ a family of lower semicontinuous convex functionals on $X$ with values in $(-\infty,+\infty]$. If for any finite set of indices $\nu_{1}, \ldots, \nu_{n}$ and for any non-negative numbers $\lambda_{1}, \ldots, \lambda_{n}$ with $\sum_{i=1}^{n} \lambda_{i}=1$, there is a $y \in X$ such that

$$
\sum_{i=1}^{n} \lambda_{i} f_{\nu_{i}}(y) \leq 0,
$$
\end{abstract}

then there exists an $x \in X$ such that $f_{\nu}(x) \leq 0, \nu \in I$.

1991 Mathematics Subject Classification: Primary 46A22.

Key words and phrases: the Hahn-Banach theorem, convex functions. 
LEMma 2. If $p$ is a convex function on a real linear space $E$ and $x_{0} \in E$, than there are a linear functional $f$ and $c \in \mathbb{R}$ such that

$$
\begin{aligned}
c+f(x) & \leq p(x) \quad \text { for } x \in E, \\
c+f\left(x_{0}\right) & =p\left(x_{0}\right) .
\end{aligned}
$$

Lemma 2 is a special case of the Hahn-Banach theorem. In [2] an analogue of Lemma 2 for sublinear functions was proved using the MarkovKakutani fixed-point theorem. Our lemma can be derived e.g. from Corollary 11.2 , p. 91 of [1].

Z. Kominek ([3], Lemma 1) has obtained a more general result (for a midpoint convex functional on a non-empty algebraically open and convex subset).

Using the above lemmas we obtain the following

THEOREM. Let $C$ be a non-empty convex subset of a real linear space $E$ and let $p: E \rightarrow \mathbb{R}$ be a convex function. If $f: C \rightarrow \mathbb{R}$ is a concave function satisfying

$$
f(x) \leq p(x) \quad \text { for } x \in C,
$$

then there exists a linear function $g: E \rightarrow \mathbb{R}$ and a constant $a \in \mathbb{R}$ such that

$$
\begin{array}{ll}
g(x)+a \leq p(x) & \text { for } x \in E, \\
f(x) \leq g(x)+a & \text { for } x \in C .
\end{array}
$$

Proof. First assume that $0 \in C$. Let $F$ be the linear topological space $\mathbb{R}^{E}$ with the Tikhonov topology. Then define

$$
\begin{aligned}
J(E) & :=\left\{g: E \rightarrow \mathbb{R}: g\left(\frac{x+y}{2}\right)=\frac{1}{2}[g(x)+g(y)], x, y \in E\right\} \\
B & :=\{g \in J(E): g \leq p \text { on } E\}, \\
B_{n} & :=\{g \in B: g(0) \geq p(0)-n\} \quad \text { for } n \in \mathbb{N} .
\end{aligned}
$$

By Lemma $2, B$ is non-empty. We also have $B=\bigcup_{n=1}^{\infty} B_{n}, B_{n} \subset B_{n+1}$ and the $B_{n}$ are convex and closed in $F$ for all $n \in \mathbb{N}$.

For each $y \in E$ we have

$$
p(0)=p\left(\frac{y-y}{2}\right) \leq \frac{1}{2}[p(y)+p(-y)],
$$

whence

$$
2 p(0)-p(-y) \leq p(y) .
$$

Consequently, for every $n \in \mathbb{N}$ the set

$$
X_{n}:=\prod_{y \in E}[2 p(0)-p(-y)-2 n, p(y)]
$$

is non-empty, convex and compact in $F$. 
We also have $B_{n} \subset X_{n}$ for $n \in \mathbb{N}$. Indeed, each $g \in B_{n}$ satisfies

$$
\begin{gathered}
g(0) \geq p(0)-n, \\
2 g(0)=g(y)+g(-y), \quad y \in E, \\
g(y) \leq p(y), \quad y \in E .
\end{gathered}
$$

Hence for every $y \in E$

$$
2 p(0)-2 n-p(-y) \leq 2 g(0)-g(-y)=g(y) \leq p(y) .
$$

Now for every $n \in \mathbb{N}$ we define

$$
C_{n}:=\{x \in C: 2 f(x)-p(2 x) \geq p(0)-n\} .
$$

It is easy to see that $C=\bigcup_{n=1}^{\infty} C_{n}$, the $C_{n}$ are convex and $C_{n} \subset C_{n+1}$ for $n \in \mathbb{N}$.

Take $x \in C_{n}$. By Lemma 2 there exists $g \in J(E)$ such that $g \leq p$ on $E$ and $g(x)=p(x)$. Then

$$
\begin{aligned}
f(x) & \leq p(x)=g(x)=g\left(\frac{2 x+0}{2}\right) \\
& =\frac{1}{2}[g(2 x)+g(0)] \leq \frac{1}{2}[p(2 x)+g(0)],
\end{aligned}
$$

whence

$$
g(0) \geq 2 f(x)-p(2 x) \geq p(0)-n .
$$

This means that $g \in B_{n}$; that is, for every $x \in C_{n}$ there exists $g \in B_{n}$ such that $g(x)=p(x)$. In particular, $g(y) \leq p(y)$ for all $y \in E$.

Fix $n \in \mathbb{N}$ for which $0 \in C_{n}$. Define $G_{x}: B_{n} \rightarrow \mathbb{R}$ for $x \in C_{n}$ by

$$
G_{x}(g)=f(x)-g(x) \quad \text { for } g \in B_{n} .
$$

It is easy to show that each $G_{x}$ is convex. Moreover, it is lower semicontinuous, for if $c \in \mathbb{R}$ and $\Pi_{x}(g)=g(x)$ for $g \in F$, then

$$
\begin{aligned}
\left\{g \in B_{n}: G_{x}(g)>c\right\} & =\left\{g \in B_{n}: g(x)<f(x)-c\right\} \\
& =\left\{g \in B_{n}: \Pi_{x}(g)<f(x)-c\right\} \\
& =B_{n} \cap \Pi_{x}^{-1}((-\infty, f(x)-c)) .
\end{aligned}
$$

The last set is open in $B_{n}$ in the Tikhonov topology.

Let $x_{1}, \ldots, x_{m} \in C_{n}$ and let $\lambda_{1}, \ldots, \lambda_{m} \geq 0$ be such that $\sum_{i=1}^{m} \lambda_{i}=1$. Put $z:=\sum_{i=1}^{m} \lambda_{i} x_{i}$. Then there exists $g \in B_{n}$ for which $g(x) \leq p(x), x \in E$, and $g(z)=p(z)$. Moreover, we have

$$
\begin{aligned}
\sum_{i=1}^{m} \lambda_{i} G_{x_{i}}(g) & =\sum_{i=1}^{m} \lambda_{i} f\left(x_{i}\right)-\sum_{i=1}^{m} \lambda_{i} g\left(x_{i}\right) \leq f\left(\sum_{i=1}^{m} \lambda_{i} x_{i}\right)-g\left(\sum_{i=1}^{m} \lambda_{i} x_{i}\right) \\
& =f(z)-g(z) \leq f(z)-p(z) \leq 0 .
\end{aligned}
$$


Hence by virtue of Lemma 1 there exists $g_{n} \in B_{n}$ such that $G_{x}\left(g_{n}\right) \leq 0$ for all $x \in C_{n}$, i.e.,

We put

$$
f \leq g_{n} \quad \text { on } C_{n}, \quad g_{n} \leq p \quad \text { on } E .
$$

$$
X:=\prod_{x \in E}[2 f(0)-p(-x), p(x)] \cap J(E) .
$$

This set is compact, convex and non-empty. In fact, we have

$$
f(0) \leq g_{n}(0)=\frac{g_{n}(x)+g_{n}(-x)}{2} \leq \frac{g_{n}(x)+p(-x)}{2},
$$

hence $2 f(0)-p(-x) \leq g_{n}(x) \leq p(x)$, whence $g_{n} \in X$

Now consider the functions $G_{x}: X \rightarrow \mathbb{R}, x \in C$, defined by

$$
G_{x}(g)=f(x)-g(x), \quad g \in X .
$$

Fix arbitrary $x_{1}, \ldots, x_{m} \in C$ and $\lambda_{1}, \ldots, \lambda_{m} \geq 0$ with $\sum_{i=1}^{m} \lambda_{i}=1$. For sufficiently large $n \in \mathbb{N}$ we have $0, x_{1}, \ldots, x_{m} \in C_{n}$ and we can find $g_{n} \in X$ such that $g_{n} \leq p$ on $E$ and $g_{n} \geq f$ on $C_{n}$. Consequently,

$$
\begin{aligned}
\sum_{i=1}^{m} \lambda_{i} G_{x_{i}}\left(g_{n}\right) & =\sum_{i=1}^{m} \lambda_{i} f\left(x_{i}\right)-\sum_{i=1}^{m} \lambda_{i} g_{n}\left(x_{i}\right) \\
& \leq f\left(\sum_{i=1}^{m} \lambda_{i} x_{i}\right)-g_{n}\left(\sum_{i=1}^{m} \lambda_{i} x_{i}\right) \leq 0 .
\end{aligned}
$$

By Lemma 1 again there exists $g_{0} \in X$ such that

$$
\begin{aligned}
g_{0}(x) \leq p(x) & \text { for } x \in E, \\
f(x) \leq g_{0}(x) & \text { for } x \in C .
\end{aligned}
$$

It is not difficult to see that there are a linear functional $g: E \rightarrow \mathbb{R}$ and a constant $a \in \mathbb{R}$ such that

$$
g_{0}(x)=g(x)+a \quad \text { for } x \in E .
$$

This ends the first part of the proof.

Now suppose that $0 \notin C$ and take an arbitrary $x_{0} \in C$. Let $C_{1}:=C-x_{0}$ and define $f_{1}: C_{1} \rightarrow \mathbb{R}$ and $p_{1}: E \rightarrow \mathbb{R}$ by

$$
\begin{array}{ll}
f_{1}(x):=f\left(x+x_{0}\right) & \text { for } x \in C_{1}, \\
p_{1}(x):=p\left(x+x_{0}\right) & \text { for } x \in E .
\end{array}
$$

It is easily seen that $f_{1}$ is concave, $p_{1}$ is convex and

$$
f_{1}(x) \leq p_{1}(x), \quad x \in C_{1} .
$$

Then there exists a linear function $g: E \rightarrow \mathbb{R}$ and a constant $c \in \mathbb{R}$ such that

$$
f_{1}(x) \leq g(x)+c, \quad x \in C_{1},
$$




$$
g(x)+c \leq p_{1}(x), \quad x \in E .
$$

Consequently,

$$
\begin{array}{ll}
f(x) \leq g(x)-g\left(x_{0}\right)+c, & x \in C, \\
g(x)-g\left(x_{0}\right)+c \leq p(x), & x \in E .
\end{array}
$$

Setting $a:=c-g\left(x_{0}\right)$ completes the proof.

Remark. It is easy to check that Theorem 1 of [2] can be obtained as a corollary to ours.

\section{References}

[1] A. Alexiewicz, Functional Analysis, Monografie Mat. 49, PWN, Warszawa 1969 (in Polish).

[2] N. Hirano, H. Komiya and W. Takahashi, A generalization of the Hahn-Banach theorem, J. Math. Anal. Appl. 88 (1982), 333-340.

[3] Z. Kominek, On additive and convex functionals, Rad. Mat. 3 (1987), 267-279.

[4] H. König, On the abstract Hahn-Banach theorem due to Rodé, Aequationes Math. 34 (1987), 89-95.

[5] K. Nikodem, On the support of midconvex operators, ibid. 42 (1991), 182-189.

[6] G. Rodé, Eine abstrakte Version des Satzes von Hahn-Banach, Arch. Math. (Basel) 31 (1978), 474-481.

INSTITUTE OF MATHEMATICS

PEDAGOGICAL UNIVERSITY

PODCHORĄŻYCH 2

30-084 KRAKÓW, POLAND 